\title{
Pilot Design for Sparse Channel Estimation in Large-Scale MIMO-OFDM System
}

\author{
Chao Xu, Jianhua Zhang, Mengmeng Liu, and Changchuan Yin \\ Key Laboratory of Universal Wireless Communications, Ministry of Education, Beijing University of Posts and Telecommunications, \\ Beijing 100876, China \\ Correspondence should be addressed to Chao Xu; xuchao_19850921@126.com
}

Received 8 January 2016; Revised 24 March 2016; Accepted 20 April 2016

Academic Editor: Larbi Talbi

Copyright (C) 2016 Chao Xu et al. This is an open access article distributed under the Creative Commons Attribution License, which permits unrestricted use, distribution, and reproduction in any medium, provided the original work is properly cited.

The pilot design problem in large-scale multi-input-multioutput orthogonal frequency division multiplexing (MIMO-OFDM) system is investigated from the perspective of compressed sensing (CS). According to the CS theory, the success probability of estimation is dependent on the mutual coherence of the reconstruction matrix. Specifically, the smaller the mutual coherence is, the higher the success probability is. Based on this conclusion, this paper proposes a pilot design algorithm based on alternating projection and obtains a nonorthogonal pilot pattern. Simulation results show that applying the proposed pattern gives the better performance compared to applying conventional orthogonal one in terms of normalized mean square error (NMSE) of the channel estimate. Moreover, the bit error rate (BER) performance of the large-scale MIMO-OFDM system is improved.

\section{Introduction}

Over the past decade, multiple input multiple output (MIMO) wireless communication has gained great popularity due to its substantial capabilities of improving the transmission rate and reliability [1]. With the $8 \times 8 \mathrm{MIMO}$ transmission scheme being incorporated into the longterm evolution advanced (LTE-A) standard, recent research efforts have been devoted to MIMO systems using more antennas which further improve transmission performance. Inspired by this, large-scale MIMO systems equipped with many antenna elements are of great research interest and considered one of the key enabling technologies for future broadband wireless communications [2].

In MIMO system, channel estimation is of crucial importance to the performance of coherent demodulation. It also helps to obtain channel state information (CSI) to support precoding and resource allocation [3]. However, when the number of antennas becomes large-scale, the conventional channel estimation methods such as the least squares (LS) and the linear minimal mean square error (LMMSE) need a mass of pilots, which seriously waste the bandwidth resources. By taking the inherent sparsity of the wireless channel into account, the compressed sensing (CS) channel estimation methods $[4,5]$ depend on much less pilots than conventional estimation methods. So, in large-scale MIMO system, this kind of channel estimation obtains more attention $[6,7]$. Now, the key challenge of using CS in channel estimation of large-scale MIMO systems lies in the following aspects: (1) seeking the sparsest representation of CSI and (2) designing the optimal pilot pattern to ensure estimation success. Our work concentrates on the latter one and tries to seek an optimal pilot pattern.

As early as the year of 2011, papers [8-10] studied the pilot design problem in CS channel estimation of single input single output (SISO) system and made an explicit research direction, that is, minimizing the mutual coherence of the reconstruction matrix. For the MIMO system, paper [11] proposed a random generation method and paper [12] puts forward two outperforming methods: the first is to minimize the largest element in the mutual coherence set whose elements are the values of mutual coherence corresponding to the pilot patterns for all multiple antennas; the second obtained a pattern in which the pilot of each antenna is orthogonal with others based on genetic algorithm (GA) and shifting mechanism. However, all the three methods above are essentially about the 
pilot location optimization. And the pilot value optimization is not considered although it is intuitively reasonable that the better pilot pattern can be obtained through joint optimization of pilot location and value. In this paper, inspired by the papers [13-15] which all study how to optimize the measurement matrix of signal reconstruction based on CS, a pilot value optimization method is proposed. Specifically, the main contributions of this paper are listed as follows:

(1) Studying an alternating projection method denoted in [13] and solving the proprietary matrix projection problem in MIMO-OFDM system;

(2) Using the alternating projection method to obtain a nonorthogonal pilot pattern in MIMO-OFDM system;

(3) Proposing a pilot design algorithm based on alternating projection and grouping shifting mechanism for CS channel estimation in large-scale MIMO-OFDM system.

The reminder of this paper is organized as follows. Section 2 gives the channel estimation model and the optimization target of the pilot design. The proposed pilot design algorithm is presented in Section 3. Simulation results are provided in Section 4. Finally, conclusions are drawn in Section 5.

For the notations used in the paper, boldface uppercase and boldface lowercase letters separately represent matrices and column vectors, while $(\cdot)^{T},(\cdot)^{*},(\cdot)^{H}, \operatorname{diag}(\cdot)$, and $\operatorname{tr}(\cdot)$ denote the transpose, the conjugate, the Hermitian transpose, the diagonal matrices, and the trace of the matrix, respectively. Finally, $\|\cdot\|_{p}$ and $\|\cdot\|_{F}$ represent the $p$-order norm and the Frobenius norm of “.”, respectively.

\section{Problem Statement}

2.1. Channel Estimation Model. Consider an OFDM downlink transmission where the base station and the terminal are equipped with $N_{t}$ transmitting antennas and one single receiving antenna, respectively. The wireless channel between the $i$ th transmitter and the receiver is frequency-selective and has the coherence time larger than the OFDM symbol duration. And it can be modeled as $L$ length finite impulse response (FIR) filter:

$$
h_{i}(n)=\sum_{l=0}^{L-1} \alpha_{i}(l) \delta(n-l)
$$

where $\alpha_{i}(l)$ is the complex weight of the $l$ th tap and the channel impulse response (CIR) vector $\boldsymbol{\alpha}_{i}=\left[\alpha_{i}(0), \alpha_{i}(1), \ldots\right.$, $\left.\alpha_{i}(L-1)\right]^{T}$ has only $K$ nonzero elements and is $K$-sparse.

Assume that the MIMO-OFDM system has $N$ subcarriers, among which $P$ subcarriers are reserved for pilots, the cyclic prefix has the length, $L_{\mathrm{cp}}$, not less than $L$, the parallel stream of $i$ th transmitter, $\left\{x_{i}(n)\right\}, n \in \boldsymbol{\Omega}=\{1,2, \ldots, N\}$, is modulated with Inverse Fast Fourier Transform (IFFT), and, within the stream, the pilot location index set is $\Lambda=$ $\left\{k_{1}, k_{2}, \ldots, k_{P}\right\} \subset \Omega$.
Let $\mathbf{X}_{i}=\operatorname{diag}\left\{x_{i}(1), x_{i}(2), \ldots, x_{i}(N)\right\}$; then the received signal samples in one OFDM symbol after FFT, $\mathbf{r} \in \mathbb{C}^{N}$, can be represented as follows:

$$
\mathbf{r}=\sum_{i=1}^{N_{t}} \mathbf{X}_{i} \mathbf{W} \boldsymbol{\alpha}_{i}+\mathbf{v}=\left[\mathbf{X}_{1} \mathbf{W}\left|\mathbf{X}_{2} \mathbf{W}\right| \cdots \mid \mathbf{X}_{N_{t}} \mathbf{W}\right] \boldsymbol{\alpha}+\mathbf{v}
$$

where $\mathbf{v}$ is the additive white Gaussian noise (AWGN) at the receiver distributed with $\mathscr{C} \mathscr{N}\left(0, \sigma^{2} \mathbf{I}_{N}\right)$, W denotes the partial FFT matrix composed of only the first $L$ columns in the standard $N$-order FFT matrix, and all the CIR vectors stack to an aggregate vector $\boldsymbol{\alpha}=\left[\boldsymbol{\alpha}_{1}^{T}, \boldsymbol{\alpha}_{2}^{T}, \ldots, \boldsymbol{\alpha}_{N_{t}}^{T}\right]^{T}$.

Let $\mathbf{X}_{P i}=\operatorname{diag}\left\{x_{i}\left(k_{1}\right), x_{i}\left(k_{2}\right), \ldots, x_{i}\left(k_{P}\right)\right\}$, and the $k_{1}$ th, $k_{2}$ th, .., $k_{P}$ th rows in $\mathbf{W}$ form the $\mathbf{W}_{P}$. Consequently, the received pilots $\mathbf{r}_{P} \in \mathbb{C}^{P}$ can be expressed as follows:

$$
\mathbf{r}_{P}=\mathbf{P} \boldsymbol{\alpha}+\mathbf{v}_{P}
$$

where $\mathbf{P}=\left[\mathbf{X}_{P 1} \mathbf{W}_{P}\left|\mathbf{X}_{P 2} \mathbf{W}_{P}\right| \cdots \mid \mathbf{X}_{P N_{t}} \mathbf{W}_{P}\right]$ denotes the reconstruction matrix and $\mathbf{v}_{P} \sim \mathscr{C} \mathscr{N}\left(0, \sigma^{2} \mathbf{I}_{P}\right)$.

Notably, for received pilots model (3), when the number of antennas is large, the number of pilots $P$ may be smaller than $L^{\prime}=L N_{t}$, the length of the aggregate channel vector, which leads the reconstruction matrix to be undetermined. As a result, it is infeasible to obtain a unique solution of $\boldsymbol{\alpha}$ by the conventional channel estimation methods. However, since $\boldsymbol{\alpha}$ is $K^{\prime}=K N_{t}$-sparse, estimating $\boldsymbol{\alpha}$ in (3) can be seen as a typical sparse signal reconstruction problem which can be solved in the framework of the CS theory. And, according to this theory, $\boldsymbol{\alpha}$ can be recovered from $\mathbf{r}_{P}$ and the deliberately designing reconstruction matrix $\mathbf{P}$ by solving the $l_{1}$-minimization problem [9]:

$$
\begin{aligned}
\widehat{\boldsymbol{\alpha}}=\arg \min & \|\boldsymbol{\alpha}\|_{1} \\
\text { s.t. } & \left\|\mathbf{r}_{P}-\mathbf{P} \boldsymbol{\alpha}\right\|_{2} \leq \beta,
\end{aligned}
$$

where $\beta$ denotes the error tolerance of reconstruction. At present, many approaches, that is, orthogonal matching pursuit (OMP) and basis pursuit (BP), have been proposed to solve this problem.

2.2. Mutual Coherence. Now, for the mutual coherence $\mu(\mathbf{P})$ of reconstruction matrix $\mathbf{P}$, there are two types of definition: one is the maximum absolute value of normalized inner products between every two columns in $\mathbf{P}$ [4] as follows:

$$
\mu_{\max }=\max _{1 \leq i, j \leq L^{\prime}, i \neq j} \frac{\left|\mathbf{p}_{i}^{H} \mathbf{p}_{j}\right|}{\left\|\mathbf{p}_{i}\right\|_{2} \cdot\left\|\mathbf{p}_{j}\right\|_{2}},
$$

where $\mathbf{p}_{i}$ represents the $i$ th column of $\mathbf{P}$, and the other named as " $t$-average coherence" is put forward by Elad in paper [13], where the coherence greater than $t$ is averaged as follows:

$$
\mu_{t, \mathrm{av}}=\frac{\sum_{1 \leq i, j \leq L^{\prime}, i \neq j}\left(\left|g_{i j}\right| \geq t\right) \cdot\left|g_{i j}\right|}{\sum_{1 \leq i, j \leq L^{\prime}, i \neq j}\left(\left|g_{i j}\right| \geq t\right)},
$$

where $g_{i j}$ denotes the $i$ th row and $j$ th column element in the Gram matrix $\mathbf{G}=\widetilde{\mathbf{P}}^{H} \widetilde{\mathbf{P}}$ and $\widetilde{\mathbf{P}}$ is the column-normalized version of $\mathbf{P}$. 
Notably, in order to make these two types of definition have a unified form, in this paper, an alternating way to describe the first definition is shown as follows:

$$
\mu_{\max }=\max _{1 \leq i, j \leq L^{\prime}, i \neq j}\left|g_{i j}\right| \text {. }
$$

According to the CS theory, the success probability of estimation is highly dependent on the mutual coherence of the reconstruction matrix. Specifically, suppose that $\boldsymbol{\alpha}$ is a necessarily sparsest reconstructed signal whose sparsity satisfies the following condition:

$$
K^{\prime}=\|\boldsymbol{\alpha}\|_{0}<\frac{1}{4}\left(1+\frac{1}{\mu(\mathbf{P})}\right) .
$$

Then, when $\mathbf{r}_{P}$ and $\mathbf{P}$ are known, both OMP and BP are guaranteed to succeed in solving problem (4) [16] and the deviation of $\widehat{\boldsymbol{\alpha}}$ from $\boldsymbol{\alpha}$ can be bounded by

$$
\|\widehat{\boldsymbol{\alpha}}-\boldsymbol{\alpha}\|_{2}^{2} \leq \frac{\left(\sigma^{2}+\beta\right)^{2}}{1-\mu(\mathbf{P})\left(4 K^{\prime}-1\right)},
$$

where $\sigma^{2}$ is the variance of noise $\mathbf{v}_{P}$ in (3) and $\beta$ is not necessarily equal to $\sigma^{2}$. The aforementioned discussion indicates that, for a $K^{\prime}$-sparse vector $\boldsymbol{\alpha}$, the smaller the $\mu(\mathbf{P})$ is, the better the approximation of $\boldsymbol{\alpha}$ can be obtained. And because $\mathbf{P}$ is determined by the pilots value $\left\{\mathbf{X}_{P i}\right\}$ and the pilots location $\Lambda$, the aim of pilot designing is minimizing $\mu(\mathbf{P})$ to improve the performance of CS channel estimation, which can be described mathematically as follows:

$$
\left\{\left\{\mathbf{X}_{P i}\right\}, \boldsymbol{\Lambda}\right\}_{\text {opt }}=\arg \min _{\left\{\mathbf{X}_{P i}\right\}, \Lambda} \mu(\mathbf{P}) .
$$

Notably, in this paper, we address this problem by optimizing $\mathbf{X}_{P i}$ and fixing $\boldsymbol{\Lambda}$. The joint optimal solution can be then obtained by traversing all of the pilot location cases.

\section{Proposed Pilot Design Scheme}

According to the definition of $\mu(\mathbf{P})$, optimization problem (10) can be transformed into the question of how to construct the Gram matrix $\mathbf{G}$ with the following properties:

(i) $\mathbf{G}$ has $\mu_{\max }=\max _{1 \leq i, j \leq L^{\prime}, i \neq j}\left|g_{i j}\right|$ or $\mu_{t \text {, av }}=$ $\left(\sum_{1 \leq i, j \leq L^{\prime}, i \neq j}\left(\left|g_{i j}\right| \geq t\right) \cdot\left|g_{i j}\right|\right) / \sum_{1 \leq i, j \leq L^{\prime}, i \neq j}\left(\left|g_{i j}\right| \geq t\right)$ small enough;

(ii) $\mathbf{G}$ has a special structure that $\mathbf{G}=\widetilde{\mathbf{P}}^{H} \widetilde{\mathbf{P}}$, where $\widetilde{\mathbf{P}}$ is the column-normalized version of $\mathbf{P}=\left[\mathbf{X}_{P 1} \mathbf{W}_{P} \mid\right.$ $\left.\mathbf{X}_{P 2} \mathbf{W}_{P}|\cdots| \mathbf{X}_{P N_{t}} \mathbf{W}_{P}\right]$.

For solving this matrix construction problem, in this paper, an algorithm named alternating projection is investigated. In the following, this algorithm is introduced; then, based on this algorithm, a pilot design scheme is proposed.

3.1. Statement of Algorithm. The alternating projection algorithm which attempts to construct a matrix that satisfies the properties (i) and (ii) simultaneously can be described in Algorithm 1.

It is worth noting that Algorithm 1 is globally convergent in a weak sense from the following theorem.
Theorem 1 (see [17]). Let $\mathcal{N}$ and $\mathscr{M}$ be closed sets, one of which is bounded. Suppose that alternating projection generates a sequence of iterates $\left\{\left(\mathbf{M}_{j}, \mathbf{N}_{j}\right)\right\}, j=1,2, \ldots, J$. This sequence has at least one accumulation point:

(i) Every accumulation point $(\overline{\mathbf{M}}, \overline{\mathbf{N}})$ lies in $\mathscr{N} \cap \mathscr{M}$.

(ii) Every accumulation point satisfies

$$
\|\overline{\mathbf{M}}-\overline{\mathbf{N}}\|_{F}=\lim _{J \rightarrow \infty}\left\|\mathbf{M}_{J}-\mathbf{N}_{J}\right\|_{F} .
$$

(iii) Every accumulation point is a generalized fixed point; namely,

$$
\|\overline{\mathbf{M}}-\overline{\mathbf{N}}\|_{F}=\inf _{\mathbf{N} \in \mathscr{N}}\|\overline{\mathbf{M}}-\mathbf{N}\|_{F}=\inf _{\mathbf{M} \in \mathscr{M}}\|\mathbf{M}-\overline{\mathbf{N}}\|_{F} .
$$

Therefore, when the number of iterations, $J$, is large enough, $\mathbf{M}_{J}$ equals $\mathbf{N}_{J}$ and both of them are the matrices that satisfy the properties (i) and (ii) simultaneously.

3.2. Implementation of Algorithm. According to the discussion above, to construct the matrix $\mathbf{G}$ utilizing alternating projection algorithm, two matrix nearness problems denoted in Step (2) and Step (3) of Algorithm 1 must be solved, respectively.

(1) Find the Nearest Matrix with Property (i). Let $\mathcal{N}_{1}$ and $\mathcal{N}_{2}$ denote the matrix collections which have sufficiently small $\mu_{\max }$ and $\mu_{t, \text { av }}$, respectively. For the matrix in $\mathcal{N}_{1}$ and $\mathcal{N}_{2}$, there are two propositions as follows.

Proposition 2. In $\mathcal{N}_{1}$, the unique matrix $\mathbf{G}$ closest to an arbitrary $L^{\prime}$-order matrix $\mathbf{Z}$ has unit diagonal entries and the off-diagonal entries satisfying

$$
\widehat{g}_{i j}= \begin{cases}z_{i j}, & \left|z_{i j}\right| \leq t_{m}, \\ t_{m} \cdot \operatorname{sign}\left(z_{i j}\right), & \text { otherwise, }\end{cases}
$$

where $t_{m}=\sqrt{\left(L^{\prime}-P\right) / P\left(L^{\prime}-1\right)}$ and $\operatorname{sign}(\cdot)$ denotes the unit direction vector of “.”.

Proposition 3. In $\mathcal{N}_{2}$, the unique matrix $\mathbf{G}$ closest to an arbitrary $L^{\prime}$-order matrix $\mathbf{Z}$ has unit diagonal entries and the off-diagonal entries satisfying

$$
\hat{g}_{i j}= \begin{cases}\gamma z_{i j}, & \left|z_{i j}\right| \geq t \\ \gamma t \cdot \operatorname{sign}\left(z_{i j}\right), & t>\left|z_{i j}\right| \geq \gamma t \\ z_{i j}, & \gamma t>\left|z_{i j}\right|\end{cases}
$$

where $\gamma$ is a shrunk factor and can be chosen from the interval $(0.5,0.9)$ in practice.

For the details about these two propositions, one can refer to the papers [17] and [13], respectively.

(2) Find the Nearest Matrix with Property (ii). The matrix nearness problem in Step (3) can be expressed essentially as the following optimization question:

$$
\widehat{\mathbf{G}}=\min _{\mathbf{G} \in \mathscr{M}}\|\mathbf{G}-\mathbf{Z}\|_{F}^{2},
$$


Input:

(i) An arbitrary initial matrix $\mathbf{M}_{0}$ and the number of iterations $J$

Output:

(i) A pair of matrices $\left(\mathbf{M}_{J}, \mathbf{N}_{J}\right)$

Procedure:

(1) Initialize $j=1$.

(2) Find $\mathbf{N}_{j}$ which satisfies Property (i) and is nearest to $\mathbf{M}_{j-1}$ in Frobenius norm.

(3) Find $\mathbf{M}_{j}$ which satisfies Property (ii) and is nearest to $\mathbf{N}_{j}$ in Frobenius norm.

(4) Increment $j$.

(5) Repeat step (2) to step (4) until $j>J$.

Algorithm 1: Alternating projection algorithm.

where $\mathbf{G}$ is determined by $\boldsymbol{\Lambda}$ and $\left\{\mathbf{X}_{P i}\right\}$ which have a finite and an infinite number of possible values, respectively, through the description above. Therefore, the $\boldsymbol{\Lambda}$ has the traversability and the next research is solving the question (15) with fixed $\Lambda$, which is still difficult due to the large number of variables in the set $\left\{\mathbf{X}_{P i}\right\}$. Fortunately, it becomes easy to be solved through the following proposition.

Proposition 4. Given $L^{\prime}=N_{t} L \leq N$ and $\mathbf{W}_{P} \mathbf{W}_{P}^{H}$ equal to the unit matrix $\mathbf{I}_{P}$,

$$
\|\mathbf{G}-\mathbf{Z}\|_{F}^{2}=\xi^{2} P N_{t}^{2}-\sum_{i=1}^{N_{t}} \sum_{j=1}^{N_{t}} \sum_{n=1}^{P} x_{i n}^{*} x_{j n} \theta_{i j n}+\eta,
$$

where $\xi=\left|x_{i n}\right|^{2}, \eta=\operatorname{tr}\left(\mathbf{Z}^{H} \mathbf{Z}\right)$, and $\theta_{i j n}$ is only related to the elements in $\mathbf{Z}$.

Proof. Expand the objective function, $\|\mathbf{G}-\mathbf{Z}\|_{F}^{2}$, as follows:

$$
\begin{aligned}
\|\mathbf{G}-\mathbf{Z}\|_{F}^{2}= & \operatorname{tr}\left((\mathbf{G}-\mathbf{Z})^{H}(\mathbf{G}-\mathbf{Z})\right) \\
= & \operatorname{tr}\left(\mathbf{G}^{H} \mathbf{G}\right)+\operatorname{tr}\left(\mathbf{Z}^{H} \mathbf{Z}\right)-\operatorname{tr}\left(\mathbf{Z}^{H} \mathbf{G}\right) \\
& -\operatorname{tr}\left(\mathbf{G} \mathbf{Z}^{H}\right) .
\end{aligned}
$$

According to Appendix A,

$$
\operatorname{tr}\left(\mathbf{G}^{H} \mathbf{G}\right)=\xi^{2} P N_{t}^{2} .
$$

According to Appendix B,

$$
\operatorname{tr}\left(\mathbf{Z}^{H} \mathbf{G}\right)=\operatorname{tr}\left(\mathbf{G} \mathbf{Z}^{H}\right)=\sum_{i=1}^{N_{t}} \sum_{j=1}^{N_{t}} \sum_{n=1}^{P} x_{i n}^{*} x_{j n} Y_{i j}[n, n],
$$

where $Y_{i j}[n, n]$ is only related to $\mathbf{Z}$ and can be represented by the $\theta_{i j n}$. So, the proposition is proved.

According to Proposition 4, given $\xi=1$, both the first and the third items of (16) are constant, so original problem (15) is equivalent to the problem

$$
\max \sum_{i=1}^{N_{t}} \sum_{j=1}^{N_{t}} \sum_{n=1}^{P} x_{i n}^{*} x_{j n} \theta_{i j n} .
$$

And this problem can be converted into $P$ parallel optimization problems each of which has only $N_{t}$ variables and can be solved by the genetic algorithm (GA) easily.

From the discussion above, a pilot design algorithm is proposed in Algorithm 2.

Notably, this algorithm is based on the condition that $L^{\prime}=$ $N_{t} \times L \leq N$ and $\mathbf{W}_{P} \mathbf{W}_{P}^{H}=\mathbf{I}$. If it is not met when $N_{t}$ is large, we can firstly divide all the antennas into some groups each of which includes $N_{t}^{\prime}<N_{t}$ antennas and meets the condition, secondly apply the pilot design algorithm on one group, and then obtain the whole pilot pattern on $N_{t}$ antennas through a shifting mechanism which is similar to paper [12].

\section{Simulation Results}

In this section, we carry out three simulations to study the proposed pilot design algorithm in the CS channel estimation of large-scale MIMO-OFDM system.

4.1. The Simulation for the Proposed Algorithm. In Figures 1 and 2, the behavior of the proposed algorithm is illustrated. We assume that the channel model based on FIR filter has $L=128$ taps in which $K=4$ taps are weighted by standard complex Gaussian random variables and the others by zero, which means that the channel is 4-sparse. As for the OFDM symbol, $N=1024$ subcarriers are utilized, among which $P$ subcarriers are reserved for pilots. Notably, $P$ is related to the sparsity of $\boldsymbol{\alpha}$ and fixed to be its double in this simulation.

In Figure 1, we obtain the convergence of $\mu_{\max }$ in three cases as follows.

Case 1. Let $N_{t}=2$, so the vector $\boldsymbol{\alpha}$ has length of 256 and sparsity of 8 .

Case 2. Let $N_{t}=4$, so the vector $\boldsymbol{\alpha}$ has length of 512 and sparsity of 16 .

Case 3. Let $N_{t}=8$, so the vector $\boldsymbol{\alpha}$ has length of 1024 and sparsity of 32 .

On the whole, $\mu_{\max }$ reduces with the increasing of iteration number in all the three cases. In addition, by comparing all the three cases, it is shown that the more the antennas utilized, the smaller the $\mu_{\max }$ and the slower the convergence obtained. Specifically, in Case 1, after six iterations, $\mu_{\max }$ 
Input:

(i) An initial matrix set $\left\{\mathbf{X}_{P i}\right\}_{0}$

(ii) A partial FFT matrix $\mathbf{W}_{P}$ with $L$ columns and $P$ orthogonal rows

(iii) The number of iterations $J$

Output:

(i) The optimized matrix set $\left\{\mathbf{X}_{P i}\right\}_{\text {opt }}$

Procedure:

(1) Initialize the iterate counter $j=0$

(2) Calculate $\mathbf{M}_{j}=\widetilde{\mathbf{P}}_{j}^{H} \widetilde{\mathbf{P}}_{j}$ where $\widetilde{\mathbf{P}}_{j}=\frac{1}{\sqrt{P}}\left[\mathbf{X}_{P 1} \mathbf{W}_{P}\left|\mathbf{X}_{P 2} \mathbf{W}_{P}\right| \cdots \mid \mathbf{X}_{P N_{t}} \mathbf{W}_{P}\right]$ and $\mathbf{X}_{P .} \in\left\{\mathbf{X}_{P i}\right\}_{j}$

(3) Use (13) and (14) to find the matrix $\mathbf{N}_{j}$ which is nearest to $\mathbf{M}_{j}$ in $\mathcal{N}_{1}$ and $\mathcal{N}_{2}$ respectively

(4) Use GA algorithm to solve (15) in which $\mathbf{Z}=\mathbf{N}_{j}$

(a) Let $n=1$

(b) Use GA algorithm to solve the following problem:

$$
\max \sum_{i, j=1}^{N_{t}} x_{i n}^{*} x_{j n} \theta_{i j n},
$$

where $\left|x_{.}\right|^{2}=1$, and $\theta_{i j n}$ is only related to $\mathbf{N}_{j}$

(c) Increment $n$, repeat the step (b) to step (c) until $n=N_{t}$ and then, obtain the $\left\{\mathbf{X}_{P i}\right\}_{j+1}$

(5) Increment $j$

(6) Repeat the step (2) to step (5) until $j=J$, then $\left\{\mathbf{X}_{P i}\right\}_{\text {opt }}=\left\{\mathbf{X}_{P i}\right\}_{J}$

Algorithm 2: Pilot design algorithm based on alternating projection.

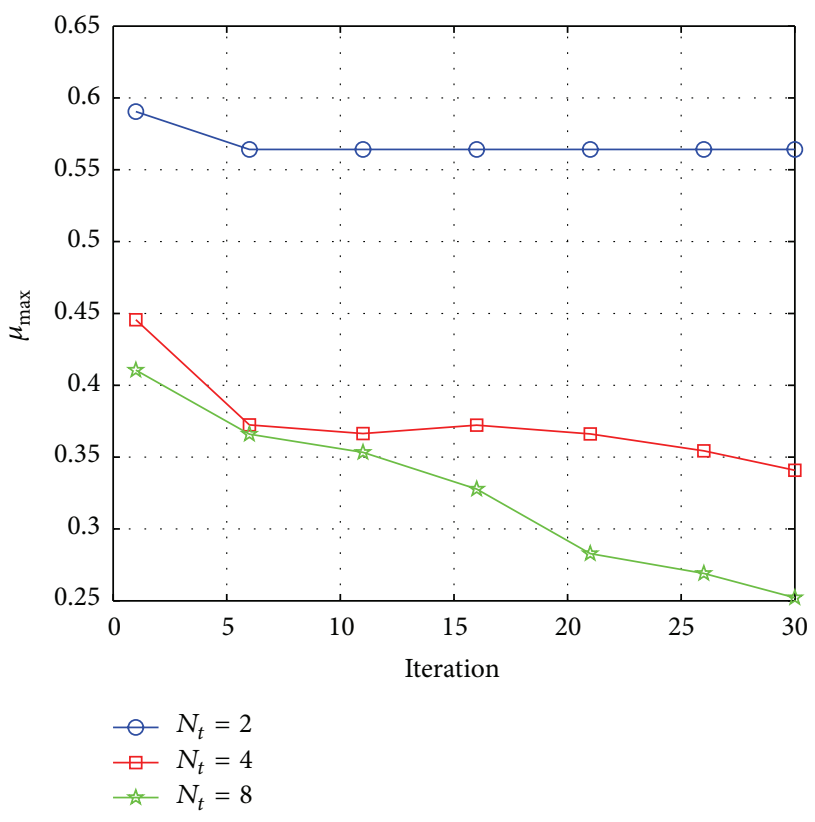

FIgURE 1: Value of $\mu_{\max }$ as a function of the iteration when $N_{t}=$ $2,4,8$.

converges to 0.56 , and, in Case 2, it is 0.35 after ten iterations. In Case $3, \mu_{\max }$ declines to 0.27 at the thirtieth iterations.

In Figure 2, we obtain the convergence of $\mu_{t}$ in the same three cases as in Figure 1. And, for each case, the dashed and solid lines are plotted when $\gamma=0.85$ and $\gamma=0.55$, respectively. As expected, $\mu_{t}$ reduces with the increasing of iteration number. In addition, the dashed and solid lines almost

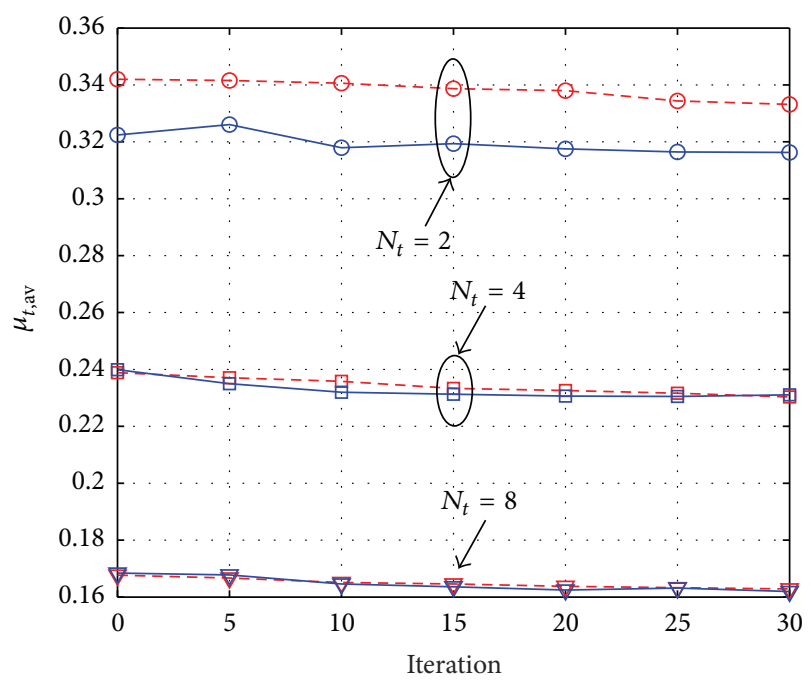

Figure 2: Value of $\mu_{t}$ as a function of the iteration when $N_{t}=2,4,8$ and $\gamma=0.85,0.55$.

overlap in Case 3, which shows that when the number of antennas increases, $\gamma$ gets smaller quite effectively.

4.2. The Simulation for the System with 4 Transmitting Antennas. By Figures 3 and 4, the performance of the proposed pilot pattern in the system is assessed. System parameters are set as $N_{t}=4, N=1024$, there are two users which configure one receiving antenna individually, and the QPSK modulation with unit amplitude is applied. The channel model used in this simulation is the same as the one in the first simulation. 


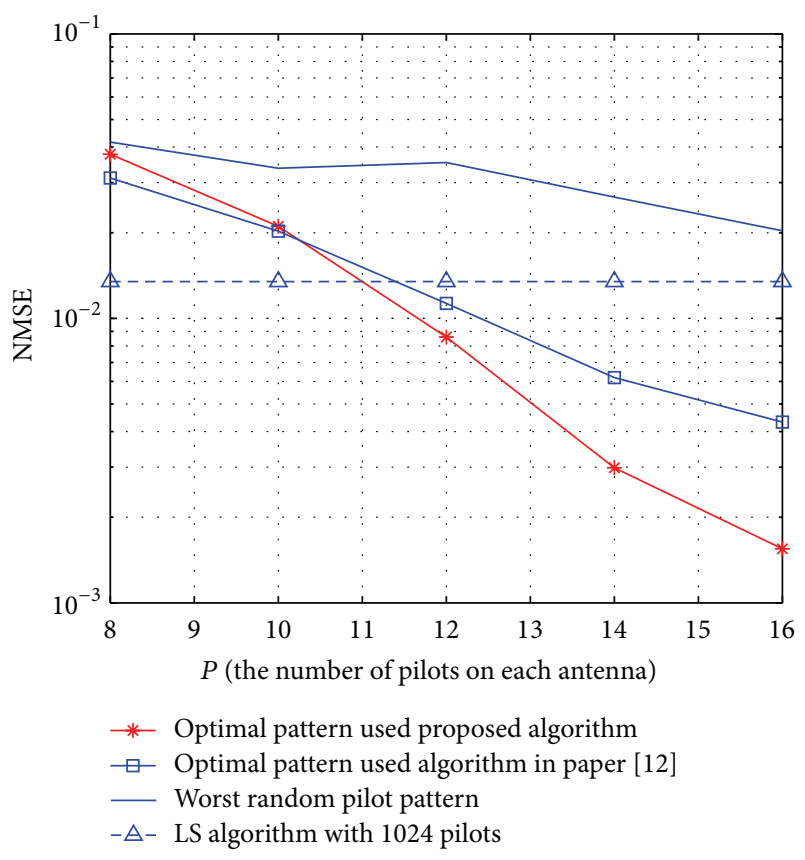

FIGURE 3: NMSE performance when SNR $=20 \mathrm{~dB}$.

For evaluating the estimation performance, the perantenna normalize mean square error (NMSE) is applied, which is computed as follows:

$$
\mathrm{NMSE}=\frac{1}{N_{\mathrm{MC}}} \sum_{n=1}^{N_{\mathrm{MC}}} \frac{\left\|\widehat{\boldsymbol{\alpha}}^{(n)}-\boldsymbol{\alpha}^{(n)}\right\|_{2}^{2}}{N_{t}\left\|\boldsymbol{\alpha}^{(n)}\right\|_{2}^{2}},
$$

where $N_{\mathrm{MC}}$ is the number of Monte Carlo iterations and $\boldsymbol{\alpha}^{(n)}$ and $\widehat{\boldsymbol{\alpha}}^{(n)}$ are the true and estimated channel vectors in the $n$th Monte Carlo iteration, respectively.

Figure 3 demonstrates the NMSE performance of three kinds of pilot pattern, that is, the worst in 100 random patterns, the patterns proposed in [12], and our proposed pattern. As a comparison, the performance of conventional LS channel estimation with all subcarriers being reserved for pilots is also shown. Notably, in order to ensure the contrast fairness, all these four patterns are made to have the equal pilot power. It is shown that as the number of pilots increases, the NMSE performance becomes better. And using the proposed pattern in this paper can obtain the best performance when the number of pilots on each antenna is more than 11.

Figure 4 illustrates the system BER performance of the four kinds of circumstance mentioned above. Similar to Figure 3, it is shown that the proposed pattern in this paper has the best performance especially when the SNR is more than $10 \mathrm{~dB}$. Notably, in the process of simulation, all subcarriers have been utilized as pilots to activate the conventional LS estimate algorithm, which causes the $100 \%$ bandwidth waste. However, in CS-based estimation, the pilots occupy only $12.5 \%$ of bandwidth. As to the computational complexity, the number of complex multiplications in LS channel estimation and CS-based channel estimation is $o\left(N L N_{t}\right)$ and $o\left(I P L N_{t}^{2}\right)$,

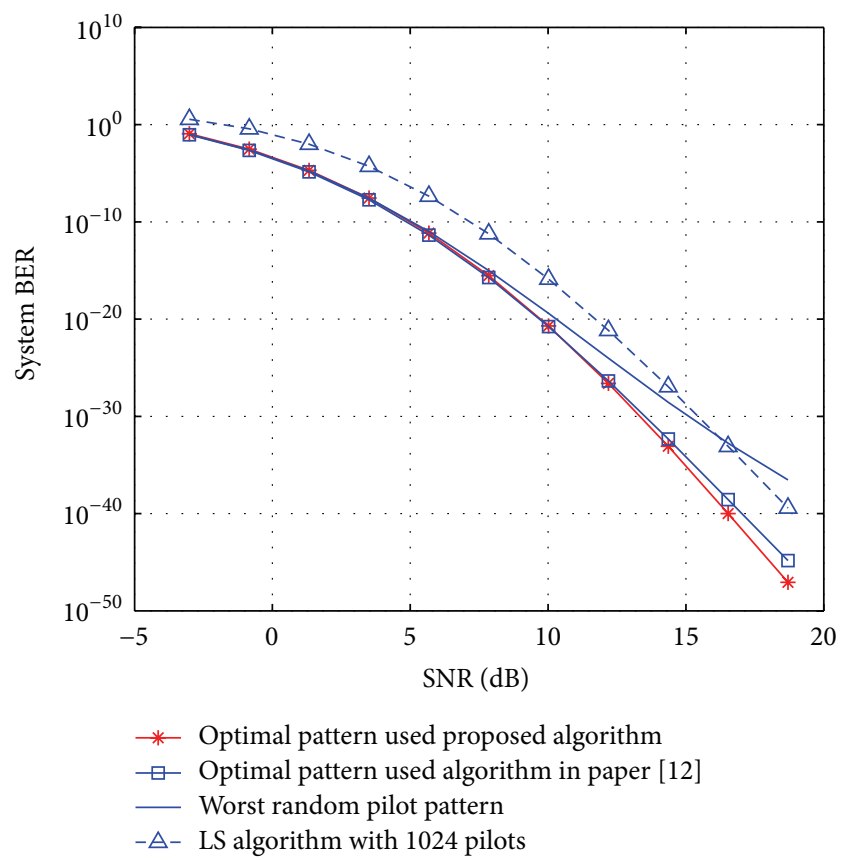

FIGURE 4: System BER when the number of pilots in each antenna is 16.

respectively, where $I$ is the number of iterations in OMP algorithm and it is approximately equal to the sparsity of vector $\boldsymbol{\alpha}$. So, the two methods of channel estimation have the same order of computational complexity. However, the CS-based channel estimation needs the sparsity of $\boldsymbol{\alpha}$ and the variance of noise as prior knowledge, which is not needed in LS channel estimation. Consider

$$
\begin{aligned}
\mathbf{P}^{H} \mathbf{P}= & {\left[\begin{array}{lll}
\mathbf{W}_{P}^{H} & & \\
& \ddots & \\
& & \mathbf{W}_{P}^{H}
\end{array}\right] \cdot\left[\begin{array}{ccc}
\mathbf{B}_{11} & \cdots & \mathbf{B}_{1 N_{t}} \\
\vdots & \ddots & \vdots \\
\mathbf{B}_{N_{t} 1} & \cdots & \mathbf{B}_{N_{t} N_{t}}
\end{array}\right] } \\
& \cdot\left[\begin{array}{cccc}
\mathbf{W}_{P} & & \\
& \ddots & \\
& & \mathbf{W}_{P}
\end{array}\right]=\left[\begin{array}{ccc}
\mathbf{C}_{11} & \cdots & \mathbf{C}_{1 N_{t}} \\
\vdots & \ddots & \vdots \\
\mathbf{C}_{N_{t} 1} & \cdots & \mathbf{C}_{N_{t} N_{t}}
\end{array}\right] .
\end{aligned}
$$

4.3. The Simulation for the System with 32 Transmitting Antennas. By Figures 5 and 6, the performance of the proposed pilot pattern in the system is assessed. Let the number of transmitting antennas, $N_{t}$, be equal to 32 and the other system parameters are the same in the second simulation. Notably, when $N_{t}=32$, the conditions $L^{\prime}=N_{t} \times L \leq N$ and $P \leq L$ are not met. According to the proposed design algorithm, all the transmitting antennas need to be grouped.

Figure 5 demonstrates the NMSE performance of three cases that divide all the transmitting antennas into 4,8 , and 16 groups, respectively. As a comparison, the performance of pilot pattern proposed in paper [12] is also shown, which is equivalent to the case that divides into 32 groups. It is shown 


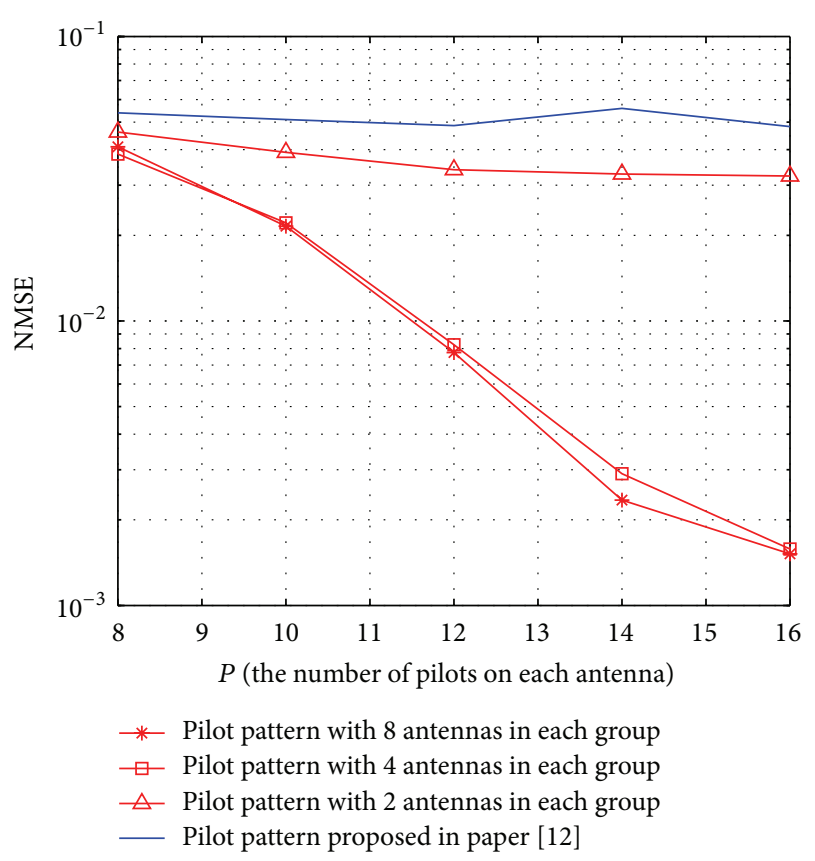

FIGURE 5: NMSE performance when SNR $=20 \mathrm{~dB}$.

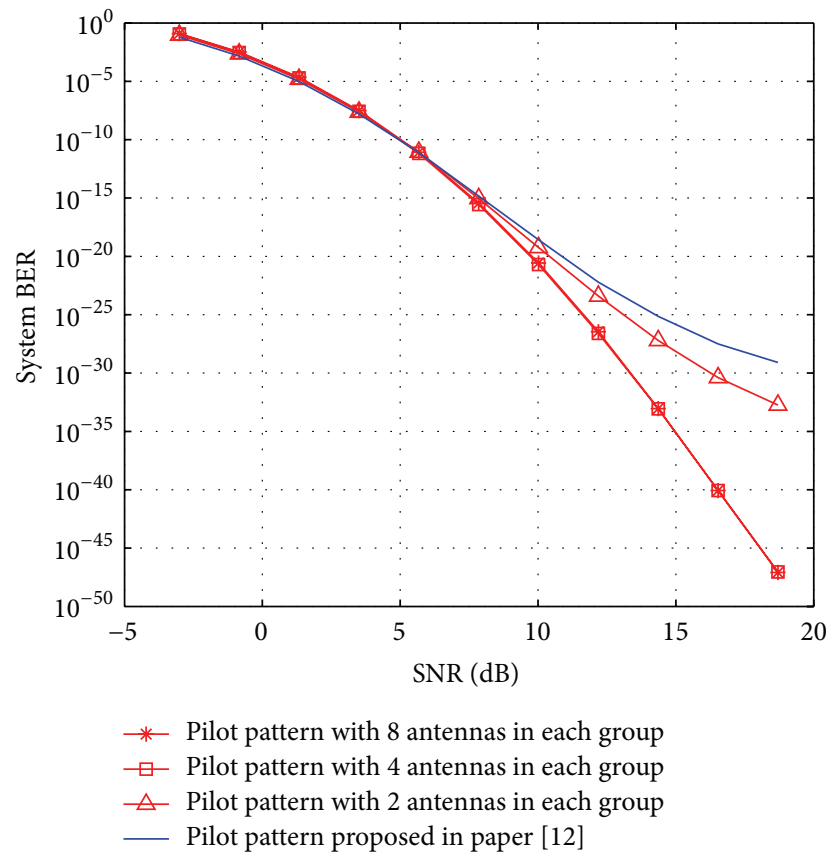

FIGURE 6: System BER when the number of pilots in each antenna is 16.

that the cases with 4 and 8 groups have the similar performance which is better than the others. And the same situation can be shown in Figure 6 which illustrates the system BER performance of the four kinds of circumstance mentioned above. According to the objective function of GA algorithm in Algorithm 2, the number of complex multiplications in the fitness function calculated process of GA algorithm is $o\left(N_{t}^{2}\right)$. Therefore, dividing 32 antennas into 8 groups is more practical because the proposed pilot design algorithm with this has the lower computation complexity.

\section{Conclusion}

In large-scale MIMO-OFDM system, the CS-based channel estimation can overcome the pilot pollution effectively because of its dramatic improving of the system spectral efficiency. Based on alternating projection, a pilot design method for the CS-based channel estimation in large-scale MIMO-OFDM system is proposed in this paper. Simulations show that the pilot pattern obtained by our method has a better performance in terms of the NMSE and BER compared to the method in [12].

\section{Appendix}

\section{A. The Derivation of $\operatorname{tr}\left(\mathbf{G}^{H} \mathbf{G}\right)$}

Define matrix B with the $i$ th row and $j$ th column block:

$$
\mathbf{B}_{i j}=\left[\begin{array}{cccc}
x_{i 1}^{*} x_{j 1} & & & \\
& x_{i 2}^{*} x_{j 2} & & \\
& & \ddots & \\
& & & x_{i P}^{*} x_{j P}
\end{array}\right] .
$$

So, $\mathbf{G}$ can be expanded as (22) and

$$
\operatorname{tr}\left(\mathbf{G}^{H} \mathbf{G}\right)=\sum_{i=1}^{N_{t}} \sum_{j=1}^{N_{t}} \operatorname{tr}\left(\mathbf{C}_{i j} \mathbf{C}_{i j}^{H}\right),
$$

where

$$
\begin{aligned}
& \mathbf{C}_{i j} \mathbf{C}_{i j}^{H}=\mathbf{W}_{P}^{H} \mathbf{B}_{i j} \mathbf{W}_{P} \mathbf{W}_{P}^{H} \mathbf{B}_{i j}^{H} \mathbf{W}_{P} \\
& =\left(V \Sigma^{H} U^{H}\right) \mathbf{B}_{i j}\left(U \Sigma V^{H}\right)\left(V \Sigma^{H} U^{H}\right) \mathbf{B}_{i j}^{H}\left(U \Sigma V^{H}\right) .
\end{aligned}
$$

Notably, the second equation is obtained by the singular value decomposition (SVD) of $\mathbf{W}_{P}$.

Because $P \leq L$ and any two rows of $\mathbf{W}_{P}$ are orthogonal, $\Sigma=[\mathbf{I}, 0]$, where $\mathbf{I}$ is a $P$-order unit matrix. Put $\Sigma$ into formula (A.3), and we can obtain that

$$
\operatorname{tr}\left\{\mathbf{C}_{i j} \mathbf{C}_{i j}^{H}\right\}=\operatorname{tr}\left\{\mathbf{B}_{i j} \mathbf{B}_{i j}^{H}\right\}=\sum_{n=1}^{P}\left|x_{i n}\right|^{2} \cdot\left|x_{j n}\right|^{2} .
$$

Because $\left|x_{i n}\right|^{2}=\xi, \forall i \in\left\{1,2, \ldots, N_{t}\right\}$, and $\forall n \in\{1,2, \ldots, P\}$,

$$
\begin{aligned}
\operatorname{tr}\left(\mathbf{G}^{H} \mathbf{G}\right) & =\sum_{i=1}^{N_{t}} \sum_{j=1}^{N_{t}} \operatorname{tr}\left(\mathbf{C}_{i j} \mathbf{C}_{i j}^{H}\right) \\
& =\sum_{i=1}^{N_{t}} \sum_{j=1}^{N_{t}} \sum_{n=1}^{P}\left|x_{i n}\right|^{2} \cdot\left|x_{j n}\right|^{2}=\xi^{2} \cdot P \cdot N_{t}^{2} .
\end{aligned}
$$

So, it is proven that $\operatorname{tr}\left(\mathbf{G}^{H} \mathbf{G}\right)$ is a constant value. 


\section{B. The Derivation of $\operatorname{tr}\left(\mathbf{Z}^{H} \mathbf{G}\right)$}

Divide the matrix $\mathbf{Z}$ into $N_{t} \times N_{t}$ blocks. The $i$ th row and $j$ th column block denoted by $\mathbf{D}_{i j}$ has the size similar to $\mathbf{C}_{i j}$ mentioned above. Then,

$$
\operatorname{tr}\left(\mathbf{Z}^{H} \mathbf{G}\right)=\operatorname{tr}\left(\mathbf{G}^{H} \mathbf{Z}\right)=\sum_{i=1}^{N_{t}} \sum_{j=1}^{N_{t}} \operatorname{tr}\left(\mathbf{C}_{i j}^{H} \mathbf{D}_{i j}\right)
$$

Expand $\operatorname{tr}\left(\mathbf{C}_{i j}^{H} \mathbf{D}_{i j}\right)$ as follows:

$$
\begin{aligned}
\operatorname{tr} & \left(\mathbf{C}_{i j}^{H} \mathbf{D}_{i j}\right)=\operatorname{tr}\left(\mathbf{W}_{P}^{H} \mathbf{B}_{i j}^{H} \mathbf{W}_{P} \mathbf{D}_{i j}\right) \\
& =\operatorname{tr}\left(\left(V \Sigma^{H} U^{H}\right) \mathbf{B}_{i j}^{H}\left(U \Sigma V^{H}\right) \mathbf{D}_{i j}\left(V V^{H}\right)\right) \\
& =\operatorname{tr}\left(\Sigma^{H} U^{H} \mathbf{B}_{i j}^{H}\left(U \Sigma V^{H}\right) \mathbf{D}_{i j} V\right) .
\end{aligned}
$$

Put $\Sigma=[\mathbf{I}, 0]$ into it, and we can obtain that

$$
\begin{aligned}
\operatorname{tr}\left\{\mathbf{C}_{i j}^{H} \mathbf{D}_{i j}\right\} & =\operatorname{tr}\left\{U^{H} \mathbf{B}_{i j}^{H} U \mathbf{X}_{i j} U^{H} U\right\} \\
& =\operatorname{tr}\left\{\mathbf{B}_{i j}^{H} U \mathbf{X}_{i j} U^{H}\right\}=\operatorname{tr}\left\{\mathbf{B}_{i j}^{H} \mathbf{Y}_{i j}\right\} \\
& =\sum_{n=1}^{P} x_{i n}^{*} x_{j n} \mathbf{Y}_{i j}[n, n],
\end{aligned}
$$

where $\mathbf{Y}_{i j}=U \mathbf{X}_{i j} U^{H}$ and $\mathbf{X}_{i j}$ where size equal to the $U$ 's is the upper left corner block of the matrix $V^{H} \mathbf{D}_{i j} V$. So, it is proven that

$$
\operatorname{tr}\left(\mathbf{G}^{H} \mathbf{Z}\right)=\sum_{i=1}^{N_{t}} \sum_{j=1}^{N_{t}} \sum_{n=1}^{P} x_{i n}^{*} x_{j n} \mathbf{Y}_{i j}[n, n] .
$$

Similarly, we can obtain that

$$
\operatorname{tr}\left(\mathbf{Z}^{H} \mathbf{G}\right)=\sum_{i=1}^{N_{t}} \sum_{j=1}^{N_{t}} \sum_{n=1}^{P} x_{i n}^{*} x_{j n} \mathbf{Y}_{i j}[n, n] .
$$

\section{Competing Interests}

The authors declare that they have no competing interests.

\section{Acknowledgments}

This research is supported in part by China Important National Science and Technology Specific Projects (2014ZX03003013), by National Key Technology Research and Development Program of China (2012BAF14B01), by National Natural Science Foundation of China (61171105 \& 61322110), and by 863 Program Project (2015AA01A703) and Doctor Funding Program (201300051100013).

\section{References}

[1] A. Paulraj, R. Nabar, and D. Gore, Introduction to Space-Time Wireless Communications, Cambridge University Press, 2003.
[2] F. Rusek, D. Persson, B. K. Lau et al., "Scaling up MIMO: opportunities and challenges with very large arrays," IEEE Signal Processing Magazine, vol. 30, no. 1, pp. 40-60, 2013.

[3] P.-L. Chiu, L.-Z. Huang, L.-W. Chai, and Y.-H. Huang, "Interpolation-based QR decomposition and channel estimation processor for MIMO-OFDM system," IEEE Transactions on Circuits and Systems. I. Regular Papers, vol. 58, no. 5, pp. 11291141, 2011.

[4] D. L. Donoho, "Compressed sensing," IEEE Transactions on Information Theory, vol. 52, no. 4, pp. 1289-1306, 2006.

[5] D. L. Donoho, M. Elad, and V. N. Temlyakov, "Stable recovery of sparse overcomplete representations in the presence of noise," IEEE Transactions on Information Theory, vol. 52, no. 1, pp. 6-18, 2006.

[6] S. Qaisar, R. M. Bilal, W. Iqbal, M. Naureen, and S. Lee, "Compressive sensing: From theory to applications, a survey," Journal of Communications and Networks, vol. 15, no. 5, pp. 443-456, 2013.

[7] W. U. Bajwa, J. Haupt, A. M. Sayeed, and R. Nowak, "Compressed channel sensing: a new approach to estimating sparse multipath channels," Proceedings of the IEEE, vol. 98, no. 6, pp. 1058-1076, 2010.

[8] L. Applebaum, W. U. Bajwa, A. R. Calderbank, J. Haupt, and R. Nowak, "Deterministic pilot sequences for sparse channel estimation in OFDM systems," in Proceedings of the 17th International Conference on Digital Signal Processing (DSP '11), pp. 1-7, IEEE, Corfu, Greece, July 2011.

[9] X. He and R. Song, "Pilot pattern optimization for compressed sensing based sparse channel estimation in OFDM systems," in Proceedings of the International Conference on Wireless Communications and Signal Processing (WCSP '10), pp. 1-5, IEEE, Suzhou, China, October 2010.

[10] C. Qi and L. Wu, "Optimized pilot placement for sparse channel estimation in OFDM systems," IEEE Signal Processing Letters, vol. 18, no. 12, pp. 749-752, 2011.

[11] C. Qi and L. Wu, "A hybrid compressed sensing algorithm for sparse channel estimation in mimo ofdm systems," in Proceedings of the IEEE International Conference on Acoustics, Speech and Signal Processing (ICASSP '11), pp. 3488-3491, Prague, Czech Republic, May 2011.

[12] X. He, R. Song, and W.-P. Zhu, "Pilot allocation for sparse channel estimation in MIMO-OFDM systems," IEEE Transactions on Circuits and Systems II: Express Briefs, vol. 60, no. 9, pp. 612-616, 2013.

[13] M. Elad, "Optimized projections for compressed sensing," IEEE Transactions on Signal Processing, vol. 55, no. 12, pp. 5695-5702, 2007.

[14] J. Xu, Y. Pi, and Z. Cao, "Optimized projection matrix for compressive sensing," EURASIP Journal on Advances in Signal Processing, vol. 2010, Article ID 560349, 2010.

[15] V. Abolghasemi, S. Ferdowsi, B. Makkiabadi, and S. Sanei, "On optimization of the measurement matrix for compressive sensing," in Proceedings of the 18th European Signal Processing Conference (EUSIPCO '10), pp. 427-431, August 2010.

[16] J. A. Tropp, I. S. Dhillon, R. Heath, and T. Strohmer, "Designing structured tight frames via an alternating projection method," ICES Report 350, 2003.

[17] J. A. Tropp, "Greed is good: algorithmic results for sparse approximation," IEEE Transactions on Information Theory, vol. 50, no. 10, pp. 2231-2242, 2004. 


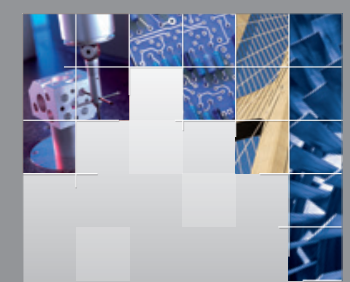

\section{Enfincering}
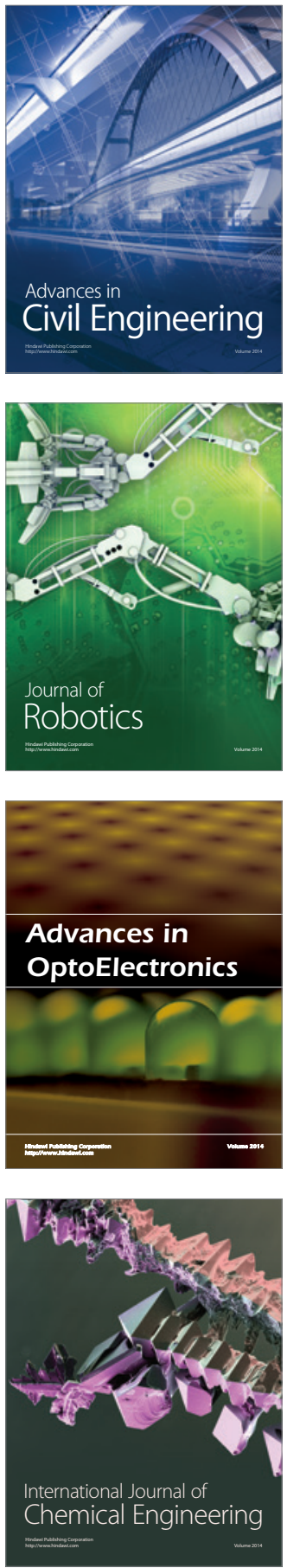

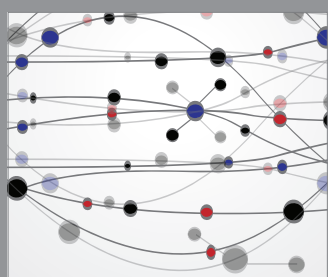

The Scientific World Journal

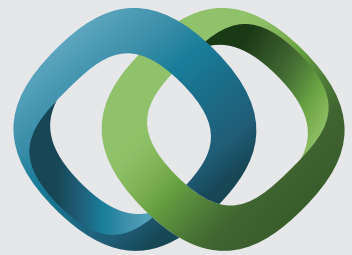

\section{Hindawi}

Submit your manuscripts at

http://www.hindawi.com

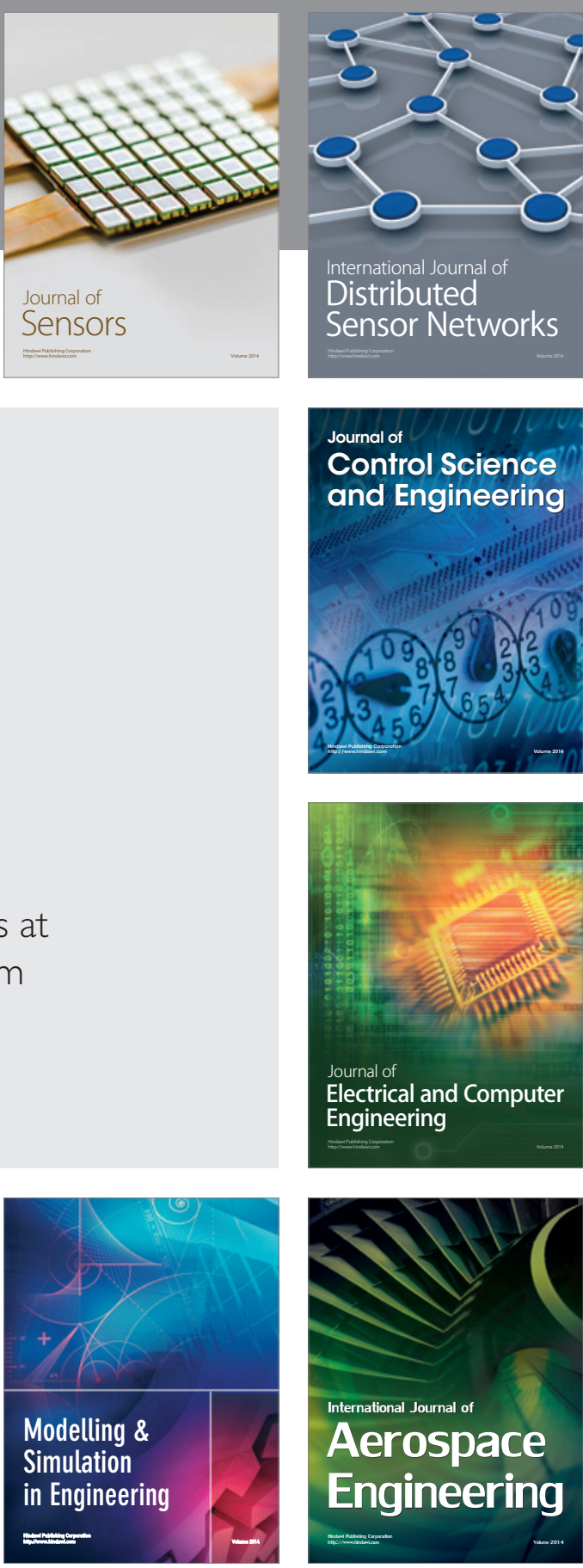

International Journal of

Distributed

Sensor Networks

Journal of

Control Science

and Engineering
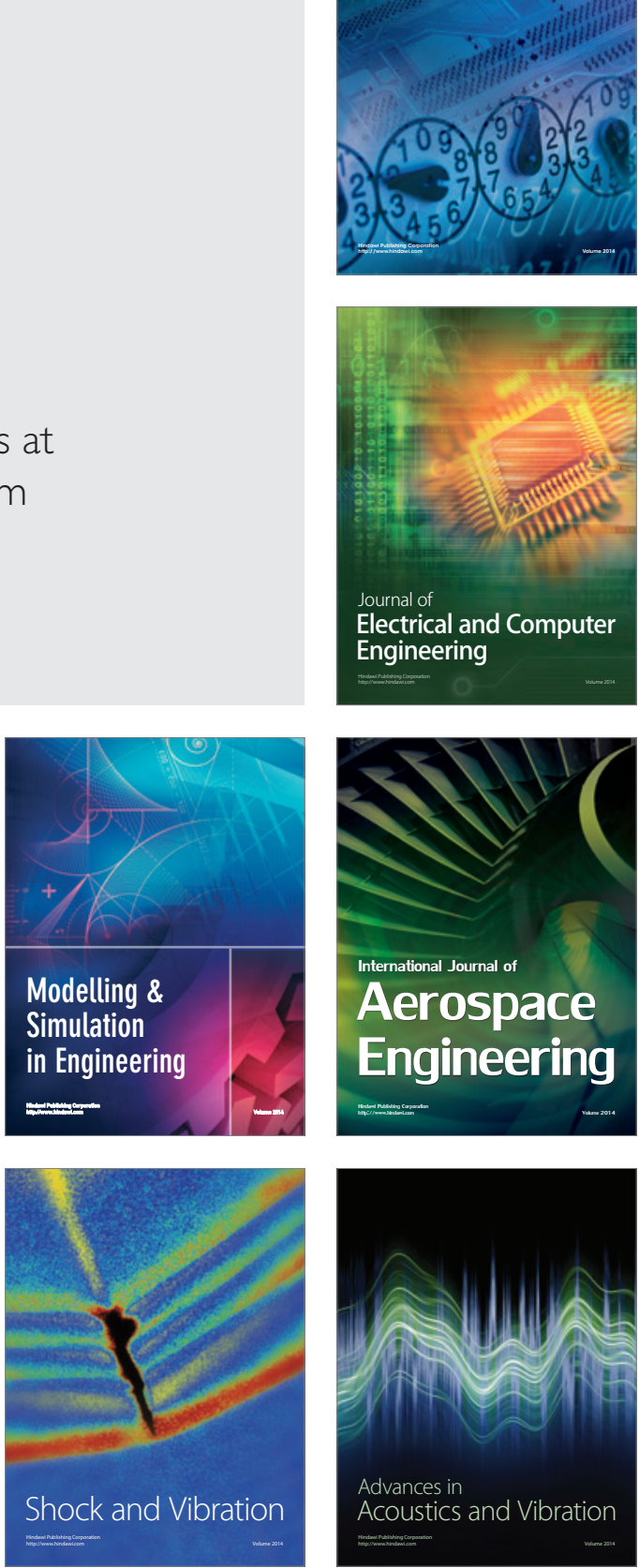